Journal of Accident and Emergency Medicine 1993 10, 43-44

\title{
Tension pneumothorax: report of two cases presenting with acute abdominal symptoms
}

\author{
G.W.HOLLINS, ${ }^{1}$ T. BEATTIE, ${ }^{1}$ I. HARPER ${ }^{2}$ \& K. LITTLE $^{2}$ \\ Departments of Accident and Emergency ${ }^{1}$ Aberdeen Royal Infirmary, Foresterhill, Aberdeen and \\ ${ }^{2}$ Royal Infirmary of Edinburgh, Lauriston Place, Edinburgh
}

\section{INTRODUCTION}

Tension pneumothorax constitutes a medical emergency and rapid diagnosis should be possible on the basis of history and clinical examination. Following treatment with the delivery of high concentration oxygen and the insertion of a large bore needle into the pleural space of the affected side, the diagnosis can be confirmed radiologically and an intercostal chest drain formally sited. ${ }^{1,2}$ We report two cases where diagnosis was not made on the basis of history and examination alone. Both cases presented with symptoms and signs suggestive of an acute intra-abdominal pathology and the diagnosis was only made on radiological grounds.

\section{CASE REPORTS}

\section{Case 1}

A 25-year-old barman presented having collapsed in the street. He gave a history of sudden onset, severe epigastric and low retrosternal chest pain. This was of stabbing quality and radiated through to the back. It was aggravated by movement and deep inspiration and there was associated dyspnoea. There were no other respiratory or gastrointestinal symptoms. There was no relevant previous medical, social or family history and he was on no regular medication.

Examination revealed him to be in severe pain. $\mathrm{He}$ was distressed and clutched his epigastrium. He was pale and sweaty with a pulse of 150 beats $\min ^{-1}$ and a blood pressure of $170 / 110 \mathrm{mmHg}$. Examination of his respiratory system revealed no abnormalities. Abdominal examination revealed rigidity with maximal tenderness in the epigastrium and bruising was also noted in this area. Bowel sounds were active.

Given the above findings, the major differential diagnoses were peptic ulcer disease or acute pancreatitis. Work-up appropriate to these diagnoses was commenced. An erect chest radiograph revealed a large pneumothorax with mediastinal shift to the left. Following drainage using a large bore needle there was immediate resolution of his symptoms and all abdominal signs. An intercostal chest drain was formally sited and full expansion of his right lung was achieved after $36 \mathrm{~h}$. He was discharged home after 3 days.

\section{Case 2}

A 37-year-old male computer operator presented with a 1-week history of general malaise associated with mild neck and back pain. On the evening of presentation he became acutely unwell with sudden onset, band-like upper abdominal pain, which radiated through to the back and was aggravated by movement and inspiration. He also complained of marked nausea but there were no other respiratory or gastrointestinal symptoms. Previous medical history included unspecified lung damage following an episode of decompression sickness 8 years previously and childhood asthma. He had been a professional diver in the North Sea until 2 years prior to presentation.

Examination revealed him to be very distressed. He was pale and sweating with a pulse of 120 beats $\mathrm{min}^{-1}$ and a blood pressure of $150 / 100 \mathrm{mmHg}$. Chest examination was normal. Examination of his abdomen revealed marked epigastric tenderness with guarding and rebound. Palpation of his left upper quadrant elicited left shoulder tip pain.

The differential diagnoses were again peptic ulcer disease and acute pancreatitis. It was only when an erect chest radiograph was performed that a large left pneumothorax with mediastinal shift to the right was noted. Following drainage with a large-bore needle there was rapid resolution of his symptoms. 
An intercostal chest drain was formally sited achieving full re-expansion of his lung and the patient was discharged after 4 days.

\section{DISCUSSION}

Various non-surgical conditions have been described which can present with severe acute abdominal pain including cardiothoracic pathology such as myocardial infarction and basal pneumonia. ${ }^{3}$ Traumatic pneumothorax resulting from lacerated lung has also been associated with intra-abdominal signs. ${ }^{4}$ However, to our knowledge, spontaneous pneumothorax has never been previously documented as presenting with acute abdominal pain.

Pneumothorax is typically associated with chest pain and dyspnoea. The pain is usually acute in onset, pleuritic in nature and reasonably well localized to the side involved. ${ }^{5}$ The pain may settle to a dull ache within hours although often it is exacerbated by exertion. Up to $80 \%$ of patients experience dyspnoea although this often settles within $24 \mathrm{~h} .^{5}$ Other minor symptoms include cough and general malaise. Occasionally patients with uncomplicated spontaneous pneumothorax may be asymptomatic.

The signs of tension pneumothorax include marked dyspnoea, cyanosis, tachycardia and hypotension. The trachea is usually deviated to the contralateral side and the affected side of the chest demonstrates increased resonance to percussion and decreased air entry and breath sounds.

The history and examination findings in these two cases led to differential diagnoses of gastrointestinal pathology. Undoubtedly one should question the examination of the chest which failed to yield any signs consistent with the ultimate diagnosis. Two recent reports have revealed that examination of the chest may be unreliable. Spiteri et al. ${ }^{6} \mathrm{com}-$ mented that their study had highlighted poor reliability in eliciting chest signs. These findings were obtained from a group of experienced chest physicians, six of whom examine for the MRCP. In the second study, performed in an accident and emergency (A\&E) setting, Dunlop et al. ${ }^{7}$ indicated that clinical signs obtained in the A\&E department correlated poorly with the ultimate diagnosis on radiograph. In conclusion we present two cases of tension pneumothorax which presented with misleading histories and examination findings. We would recommend that chest pathology, including pneumothorax, be considered in the differential "diagnosis of acute abdominal pain and consequently full and careful examination of the chest be undertaken. To delay the emergency treatment of tension pneumothorax until a chest radiograph is available could be fatal.

\section{REFERENCES}

1. American College of Surgeons (1984) Advanced Trauma and Life Support Course. Student's Manual, 74.

2. Serementis M.G. (1970) The management of spontaneous pneumothorax. Chest 57, 65-68.

3. Cuschieri A. (1988) The acute abdomen and disorders of the peritoneal cavity. In: Essential Surgical Practice 2nd edition (eds Cuschieri A., Giles G. \& Moosa A.) pp. 1233. John Wright \& Sons, Bristol.

4. Clane A. (ed.) (1986) Hamilton Bailey's Demonstration of Physical Signs in Clinical Surgery 12th edition, pp. 331. John Wright \& Sons, Bristol.

5. O'Neil S.J. (1987) Spontaneous pneumothorax: Aetiology, management and complications. Irish Medical Journal 80(11), 306-311.

6. Spiteri M.A., Cook D.G. \& Clark S.W. (1988) Reliability of eliciting physical signs in examination of the chest. Lancet 1, 873-875.

7. Dunlop M.G., Beattie T.F., Preston P.G. \& Steedman D.J. (1989) Clinical assessment an radiography following blunt chest trauma. Archives of Emergency Medicine 6(2), 125-127. 